\title{
Geometric-Process-Based Battery Management Optimizing Policy for the Electric Bus
}

\author{
Yan Li, Jin-kuan Wang, Peng Han, and Ying-hua Han \\ School of Information Science and Engineering, Northeastern University, Shenyang 110819, China \\ Correspondence should be addressed to Yan Li; liyan@mail.neuq.edu.cn
}

Received 6 May 2015; Revised 5 July 2015; Accepted 16 July 2015

Academic Editor: Xiaosong $\mathrm{Hu}$

Copyright ( 2015 Yan Li et al. This is an open access article distributed under the Creative Commons Attribution License, which permits unrestricted use, distribution, and reproduction in any medium, provided the original work is properly cited.

\begin{abstract}
With the rapid development of the electric vehicle industry and promotive policies worldwide, the electric bus (E-bus) has been adopted in many major cities around the world. One of the most important factors that restrain the widespread application of the E-bus is the high operating cost due to the deficient battery management. This paper proposes a geometric-process-based (GPbased) battery management optimizing policy which aims to minimize the average cost of the operation on the premise of meeting the required sufficient battery availability. Considering the deterioration of the battery after repeated charging and discharging, this paper constructs the model of the operation of the E-bus battery as a geometric process, and the premaintenance time has been considered with the failure repairment time to enhance the GP-based battery operation model considering the battery cannot be as good as new after the two processes. The computer simulation is carried out by adopting the proposed optimizing policy, and the result verifies the effectiveness of the policy, denoting its significant performance on the application of the E-bus battery management.
\end{abstract}

\section{Introduction}

With the intensification of the environmental pollution as well as the fast development of the smart grid and the vehicle industry, the electric vehicle (EV) will be a significant trend in the vehicle industry for its environment-friendly, energy saving, and high energy utilization rate characteristics $[1,2]$. With the massive access of the EVs in the near future, the electric bus, that is, E-bus, will constitute a significant part in the public transportation [3]. And, with the development of the battery-switching technology, the battery-switching station (BSS) is the necessary refueling infrastructure for the adoption of the E-bus $[4,5]$.

Due to the aging of the E-bus batteries through repeated charging and discharging as well as stochastic impacts of the daily usage, the state of health $(\mathrm{SOH})$ of the batteries degenerates, causing the rising of batteries failing probability. Those disadvantages above will result in the reduction of the battery life-cycle, the decline of the battery's availability, and the increasement of the E-bus daily cost-in-use. Therefore, the management of the battery should be optimized to further enhance the operating availability and reduce the long-run average cost per unit time, and many related studies have been carried out focusing on the problems of battery operation and repairment.

Gould et al. [6] propose an adaptive battery model to observe the battery voltages and monitor the degradation of the estimated dynamic model parameters, and Agarwal et al. [7] build the battery model that incorporates with the recovery effect for accurate life-cycle estimation and make a suggestion about the maximum available energy approximated at charge/discharge nominal power level.

In the state of charge (SOC) estimation, Wang and Liu [8] gather the parameters of battery voltage, current, and temperature in real time and estimate the battery state of charge as well as implementing the fault diagnosis or alarming according to the battery status to achieve the full and efficient use of battery power.

In the SOH monitoring, Li et al. [9] study the EV battery monitoring and management system based on monitoring and analyzing the performance of Li-ion battery and the battery fault in the operation of electric vehicle; Han et al. [10] propose a novel health prediction model of Li-ion battery based on sample entropy and establish the prediction 


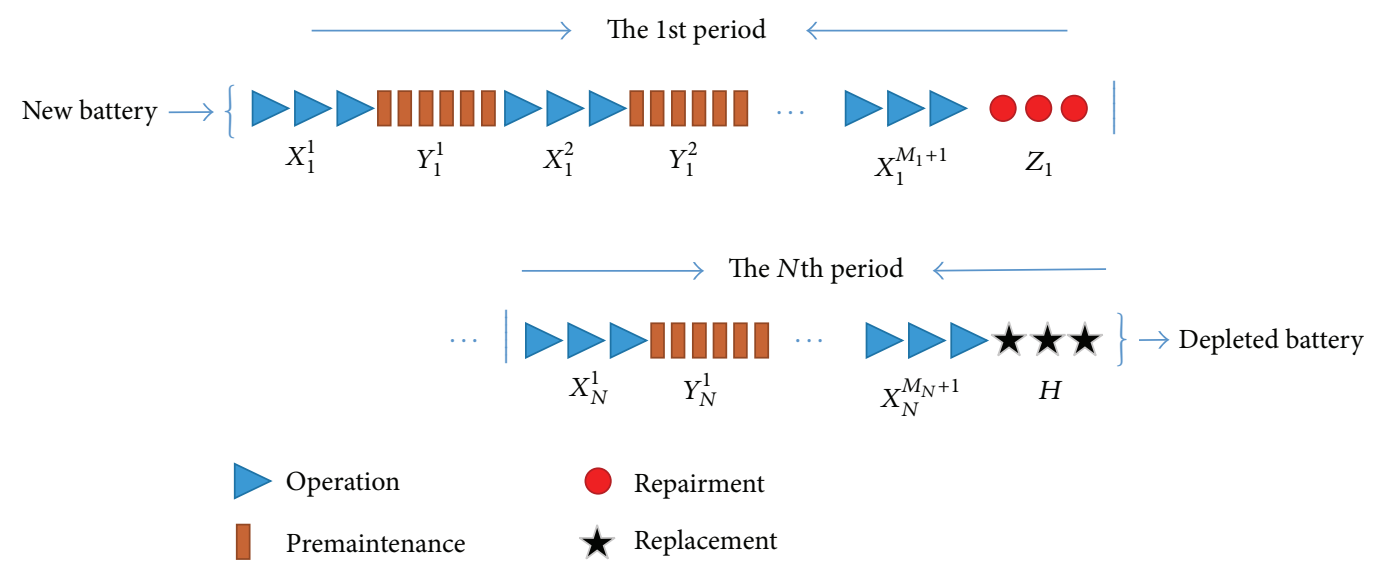

FIGURE 1: Life-cycle of the E-bus battery.

model by calculating the sample entropy, using Arrhenius formula and optimizing and fitting polynomial; Paul-Henri and Vincent [11] propose a scheme of state of charge and state of health estimation using a hybridization of Kalman filtering, Recursive Least Squares approach, and Support Vector Machines learning; and Dung et al. [12] propose a state of health estimation system based on time-constantratio measurement, which achieves the purpose of an environmental-impedance-free and fast $\mathrm{SOH}$ estimation.

However, the previous modeling, estimation, and monitoring studies related to the EV batteries mainly concentrate on the prediction of the SOC or SOH and seldom consider the effects of the battery premaintenance, which is critical in prolonging the life-cycle of the battery. And the geometricprocess-based (GP-based) method, with its practical advantages in characterizing the whole operation process of the components in a system [13], gains worldwide scholar's attention in recent years, and many researches have been carried out based on GP in different application domains. Tan et al. [14] and Jia et al. [15] study an optimal maintenance strategy for one component and present the optimal tradeoff model of cost and availability. Lam [16] introduces a geometric process $\delta$-shock maintenance model for a repairable system and adopts a replacement policy for minimizing the long-run average cost per unit time. Wang and Zhang [17-20] as well as Zhang [21] propose a GP-based preventive repairment policy to solve the efficiency for a deteriorating and valuable system to minimize the average cost rate, and Han et al. [22] present a generalized formulation for determining the optimal operating strategy and cost optimization for battery and formulate the operating strategy as a nonlinear optimization problem. Most of the studies based on GP denote a good applicability in the related fields, but their assumption that the property of the component, for example, the EV battery, after premaintenance is as good as new is not fully compatible with the E-bus battery considering its chemical reactions within. Aiming at improving the insufficient aspects mentioned above, the main research work of this paper is carried out as follows:

(1) A monotone process model of the E-bus battery is proposed by applying GP theory.
(2) The process of the premaintenance is modelled on condition that the battery, after premaintenance, cannot be as good as new.

(3) The long-run average cost per unit time is optimized on the premise of meeting the required availability under the proposed E-bus battery management optimizing policy.

The content of the paper is arranged as follows: in Section 2, the mathematical model is proposed for the E-bus battery life-cycle based on GP, and the necessary definitions and the assumptions are given; in Section 3, E-bus battery management optimizing policy is applied to estimate the long-run average cost per unit time under the constraint of the availability; in Section 4, the simulation results are evaluated for the $220 \mathrm{Ah} \mathrm{Li-ion} \mathrm{battery} \mathrm{and} \mathrm{the} \mathrm{effectiveness}$ of the proposed E-bus battery management optimizing policy is verified; and the paper concludes in Section 5.

\section{Mathematical Model}

The mathematical model of the E-bus battery life-cycle based on GP is firstly proposed. Assume that the E-bus battery is new at the beginning, and the initial state of health $(\mathrm{SOH})$ is $R_{0}$. When a single operation is completed, the E-bus battery will be premaintained. Then the maintenance policy $(M, N)$ is applied, where the amount of the premaintenance within a single failure repairment is $M$ while the failure repairment amount during the battery life-cycle is $N$.

By the definition of GP, a complete life-cycle of an E-bus battery is actually a time interval between the beginning of the battery's initiated utilization and the first replacement or a time interval between two consecutive replacements, and the life-cycle of the E-bus battery is illustrated in Figure 1. For $n=1,2, \ldots, N-1$, the time interval between the $(n-1)$ th and the $n$th failure repairment in a cycle can be defined as the $n$th period of the E-bus battery life-cycle. Given the battery operating time after the $i$ th premaintenance in the $n$th period $\left\{X_{n}^{i}, n=1,2, \ldots, i=1,2, \ldots\right\}$, the $i$ th premaintenance time in the $n$th period $\left\{Y_{n}^{i}, n=1,2, \ldots, i=1,2, \ldots\right\}$, and the failure repairment time in the $n$th period $\left\{Z_{n}, n=1,2, \ldots\right\}$, 
let $\left\{M_{n}, n=1,2, \ldots\right\}$ be the amount of the premaintenance in the $n$th period and $H$ the replacement time. Finally, the replacement policy $(M, N)$ is applied by which the battery is replaced by a new one at the time when the $\mathrm{SOH}$ is less than or equal to the threshold $R$ after the $N$ th period. And the depleted battery that has been replaced will be sold or recycled for the secondary usage.

Considering the chemical property of the battery, the battery's $\mathrm{SOH}$ will deteriorate in the process of repeated charging and discharging. The following definitions and assumptions are given.

Definition 1. The depreciation rate of the battery after a single operation is $w_{1}$, the recovery rate after a single premaintenance is $w_{2}$, and the descent rate after the failure repairment is $w_{3}$, where $\left(1+w_{2}\right)\left(1-w_{1}\right)<1$ denotes that the premaintenance process will improve the performance of the battery by reducing but not eliminating the loss of the $\mathrm{SOH}$ during the operating process.

Definition 2. The successive battery operating time $\left\{X_{n}^{i}, i=\right.$ $1,2, \ldots\}$ after premaintenance in the $n$th period forms a GP with ratio $a$ and $E\left(X_{1}^{1}\right)=\lambda$, and the premaintenance time $\left\{Y_{n}^{i}, i=1,2, \ldots\right\}$ in the $n$th period forms a GP with ratio $b$ and $E\left(Y_{1}^{1}\right)=\mu$, while the consecutive failure repairment time $\left\{Z_{n}, n=1,2, \ldots\right\}$ constitutes a GP with ratio $c$ and $E\left(Z_{1}\right)=v$. Besides, the replacement time $H$ is a random variable with $E(H)=\tau$.

Definition 3. The premaintenance cost rate is $c_{m}$, the failure repairment cost rate is $c_{f}$, the benefit rate of the battery operating is $c_{b}$, the replacement cost rate is $c_{r}$ corresponding to the replacement time $H$, the cost of the new battery is $c_{N}$, and the depleted battery recycling benefit is $c_{c}$.

Assumption 4. The processes of $\left\{X_{n}^{i}, i=1,2, \ldots\right\},\left\{Y_{n}^{i}, i=\right.$ $1,2, \ldots\}$, and $\left\{Z_{n}, n=1,2, \ldots\right\}$ are independent. Besides, $X_{n}^{i}$, $i=1,2, \ldots, M+1, n=1,2, \ldots, N$ and $Y_{n}^{i}, i=1,2, \ldots, M$, $n=1,2, \ldots, N$ are independent and identically distributed (i.i.d.) random variables.

Assumption 5. Neither the premaintenance nor the repairment can repair the battery to be as good as new.

Based on Definitions 1-3 and Assumptions 4-5, the studied policy is based on the GP model with premaintenance. The battery after premaintenance is not as good as new and the successive operating time in one period will form a GP. Furthermore, the GP model is a deteriorating system for $a>$ 1 , and it is an improving system for $b<1$ and $c<1$.

\section{E-Bus Battery Management Optimizing Policy}

The optimization of the E-bus battery management policy is to find the proper $M$ and $N$ that minimize the long-run average cost per unit time on the premise of meeting the required availability. In the defined mathematical model, the related functions are deduced.
The successive operating time with the ratio $a$ can be derived through the following function:

$$
\begin{gathered}
E\left[\sum_{i=1}^{M+1} X_{1}^{i}\right]=\sum_{i=1}^{M+1} \frac{\lambda}{a^{i}}, \\
E\left[\sum_{i=1}^{M+1} X_{2}^{i}\right]=A \sum_{i=1}^{M+1} \frac{\lambda}{a^{i}}, \\
\vdots \\
E\left[\sum_{i=1}^{M+1} X_{N}^{i}\right]=A^{N-1} \sum_{i=1}^{M+1} \frac{\lambda}{a^{i}},
\end{gathered}
$$

where $A=a^{-M}$ and $M$ is the amount of the premaintenance; then

$$
E \sum_{n=1}^{N} \sum_{i=1}^{M+1} X_{n}^{i}=\sum_{n=1}^{N}\left(E \sum_{i=1}^{M+1} X_{n}^{i}\right)=\frac{1-A^{N}}{1-A} \sum_{i=1}^{M+1} \frac{\lambda}{a^{i}}
$$

And the successive premaintenance time with the ratio $b$ can be derived through the following function:

$$
\begin{gathered}
E\left[\sum_{i=1}^{M} Y_{1}^{i}\right]=\sum_{i=1}^{M} \frac{\mu}{b^{i-1}}, \\
E\left[\sum_{i=1}^{M} Y_{2}^{i}\right]=B \sum_{i=1}^{M} \frac{\mu}{b^{i-1}}, \\
\vdots \\
E\left[\sum_{i=1}^{M} Y_{N}^{i}\right]=B^{N-1} \sum_{i=1}^{M} \frac{\mu}{b^{i-1}}, \\
E \sum_{n=1}^{N} \sum_{i=1}^{M} Y_{n}^{i}=\sum_{n=1}^{N}\left(E \sum_{i=1}^{M} Y_{n}^{i}\right)=\frac{1-B^{N}}{1-B} \sum_{i=1}^{M} \frac{\mu}{b^{i-1}},
\end{gathered}
$$

where $B=b^{1-M}$.

The long-run average cost per unit time on the premise of meeting the required availability is the major target for optimizing the E-bus battery management. For the commercial operation of the BCS, the availability of the E-bus is the ratio of the battery operation time to its total life-cycle. In the proposed policy, firstly, the long-run average cost per unit time $C(M, N)$ is defined in function (4). Consider

$$
C(M, N)=\frac{C_{\text {Sum }}}{T}
$$

where $C_{\text {Sum }}$ and $T$ are given by function (5). Consider

$$
\begin{aligned}
& C_{\text {Sum }}=E\left(c_{m} \sum_{n=1}^{N} \sum_{i=1}^{M} Y_{n}^{i}-c_{b} \sum_{n=1}^{N} \sum_{i=1}^{M+1} X_{n}^{i}+c_{f} \sum_{n=1}^{N-1} Z_{n}+c_{r} H\right. \\
& \left.\quad+c_{N}-c_{c}\right), \\
& T=E\left(\sum_{n=1}^{N} \sum_{i=1}^{M+1} X_{n}^{i}+\sum_{n=1}^{N} \sum_{i=1}^{M} Y_{n}^{i}+\sum_{n=1}^{N-1} Z_{n}+H\right) .
\end{aligned}
$$


Then,

$$
\begin{gathered}
C(M, N)=\frac{E\left(c_{m} \sum_{n=1}^{N} \sum_{i=1}^{M} Y_{n}^{i}-c_{b} \sum_{n=1}^{N} \sum_{i=1}^{M+1} X_{n}^{i}+c_{f} \sum_{n=1}^{N-1} Z_{n}+c_{r} H+c_{N}-c_{c}\right)}{E\left(\sum_{n=1}^{N} \sum_{i=1}^{M} Y_{n}^{i}+\sum_{n=1}^{N} \sum_{i=1}^{M+1} X_{n}^{i}+\sum_{n=1}^{N-1} Z_{n}+H\right)} \\
=\frac{c_{m}\left(\left(1-B^{N}\right) /(1-B)\right) \sum_{i=1}^{M}\left(\mu / b^{i-1}\right)-c_{b}\left(\left(1-A^{N}\right) /(1-A)\right) \sum_{i=1}^{M+1}\left(\lambda / a^{i}\right)+c_{f} \sum_{n=1}^{N-1}\left(v / c^{n}\right)+c_{r} \tau+c_{N}-c_{c}}{\left(\left(1-B^{N}\right) /(1-B)\right) \sum_{i=1}^{M}\left(\mu / b^{i-1}\right)+\left(\left(1-A^{N}\right) /(1-A)\right) \sum_{i=1}^{M+1}\left(\lambda / a^{i}\right)+\sum_{n=1}^{N-1}\left(v / c^{n}\right)+\tau} .
\end{gathered}
$$

According to the update process with the E-bus battery management optimizing policy, the availability $A(M, N)$, as another key constraint in the battery life-cycle, is calculated through function (7). Consider

$$
\begin{aligned}
A(M, N) & =\frac{T_{\text {Operation }}}{T}=\frac{E\left(\sum_{n=1}^{N} \sum_{i=1}^{M+1} X_{n}^{i}\right)}{E\left(\sum_{n=1}^{N} \sum_{i=1}^{M} Y_{n}^{i}+\sum_{n=1}^{N} \sum_{i=1}^{M+1} X_{n}^{i}+\sum_{n=1}^{N-1} Z_{n}+H\right)} \\
& =\frac{\left(\left(1-A^{N}\right) /(1-A)\right) \sum_{i=1}^{M+1}\left(\lambda / a^{i}\right)}{\left(\left(1-B^{N}\right) /(1-B)\right) \sum_{i=1}^{M}\left(\mu / b^{i-1}\right)+\left(\left(1-A^{N}\right) /(1-A)\right) \sum_{i=1}^{M+1}\left(\lambda / a^{i}\right)+\sum_{n=1}^{N-1}\left(v / c^{i}\right)+\tau} .
\end{aligned}
$$

Considering the deteriorating performance of the battery in the case of repeated charging and discharging, it will be replaced by a new one when its $\mathrm{SOH}$ drops to $R$, while $R(M, N)$ is correlated with the depreciation rate of the battery after single operating $w_{1}$ and the recovery rate after the premaintenance $w_{2}$. Firstly, at the end of the 1st period, $R(M, 1)$ is reduced to

$$
R(M, 1)=\left(1-w_{1}\right)^{M+1}\left(1+w_{2}\right)^{M}\left(1-w_{3}\right) R_{0} .
$$

Then, before the replacement, the $\mathrm{SOH}$ after $N$ th repairment $R(M, N)$ is shown in function (9) and is subject to $R(M, N) \geq R$. Consider

$$
\begin{aligned}
R(M, N) & =R(M, 1)^{N-1}\left(1-w_{1}\right)^{M+1}\left(1+w_{2}\right)^{M} \\
& =\left(\left(1-w_{1}\right)^{M+1}\left(1+w_{2}\right)^{M}\right)^{N}\left(1-w_{3}\right)^{N-1} \\
& =\left(1-w_{1}\right)^{N(M+1)}\left(1+w_{2}\right)^{N M}\left(1-w_{3}\right)^{N-1}
\end{aligned}
$$

To acquire the relationship between variable $M$ and $N$, take the logarithm for both sides of the inequation below:

$$
\begin{aligned}
& N(M+1) \ln \left(1-w_{1}\right)+N M \ln \left(1+w_{2}\right) \\
& \quad+(N-1) \ln \left(1-w_{3}\right) \geq \ln R, \\
& M N\left(\ln \left(1-w_{1}\right)\left(1+w_{2}\right)\right)+N \ln \left(1-w_{1}\right)\left(1-w_{3}\right) \\
& \quad-\ln \left(1-w_{3}\right) \geq \ln R, \\
& M N\left(\ln \left(1-w_{1}\right)\left(1+w_{2}\right)\right) \\
& \quad+N \ln \left(1-w_{1}\right)\left(1-w_{3}\right) \geq \ln R\left(1-w_{3}\right) ;
\end{aligned}
$$

then the relationship between variable $M$ and $N$ will be

$$
\begin{aligned}
& N \\
& =\left\lfloor\frac{\ln R\left(1-w_{3}\right)}{M\left(\ln \left(1-w_{1}\right)\left(1+w_{2}\right)\right)+\ln \left(1-w_{1}\right)\left(1-w_{3}\right)}\right\rfloor .
\end{aligned}
$$

With the certain parameter $R$ being the $\mathrm{SOH}$ of the battery at the time replacement occurred, functions (6) and (7) are the bivariate functions about $M$ and $N$. When $M$ is fixed, the bivariate functions turn into univariate functions, and the management optimizing policy $N^{*}$ is determined analytically or numerically. However, if $M$ is a variable to be determined, the optimizing about $C\left(M^{*}, N^{*}\right)$ and $A\left(M^{*}, N^{*}\right)$ needs to be set up based on the rationalized configuration of $M^{*}$ and $N^{*}$. Therefore, the objective function is to determine an optimal management policy $\left(M^{*}, N^{*}\right)$ for minimizing the long-run average cost per unit time $C(M, N)$ and limiting the availability $A(M, N)$ in a certain range, which can be expressed as

$$
\begin{aligned}
C\left(M^{*}, N^{*}\right): \min & C(M, N)=\min \left(\frac{C_{\text {Sum }}}{T}\right) \\
\text { s.t. } & A\left(M^{*}, N^{*}\right)=\frac{T_{\text {Operation }}}{T} \geq A_{0},
\end{aligned}
$$

where $A_{0}$ is the threshold of the availability that meets the minimum demand of the E-bus.

\section{Simulation Results}

The computer simulation is adopted to verify the proposed GP-based E-bus battery management optimizing policy. Originally from the $220 \mathrm{Ah} \mathrm{Li}$-ion battery, the main parameters of the GP [22] are listed in Table 1, and the key parameters of the cost in the simulation are listed in Table 2. 
TABLE 1: Parameters setting of the GP.

\begin{tabular}{lccccccccccc}
\hline Parameter & $a$ & $b$ & $c$ & $\lambda$ & $\mu$ & $v$ & $\tau$ & $w_{1}$ & $w_{2}$ & $w_{3}$ & $R_{0}$ \\
\hline Value & 1.02 & 0.995 & 0.8 & 10 & 3.5 & 9 & 5 & $0.8 \%$ & $0.7 \%$ & $1.5 \%$ & 1 \\
\hline
\end{tabular}

TABLE 2: Parameters setting of the cost.

\begin{tabular}{lcccccc}
\hline Parameter & $c_{b}$ & $c_{m}$ & $c_{f}$ & $c_{r}$ & $c_{c}$ & $c_{N}$ \\
\hline Value & 10 & 12 & 25 & 40 & 500 & 2000 \\
\hline
\end{tabular}

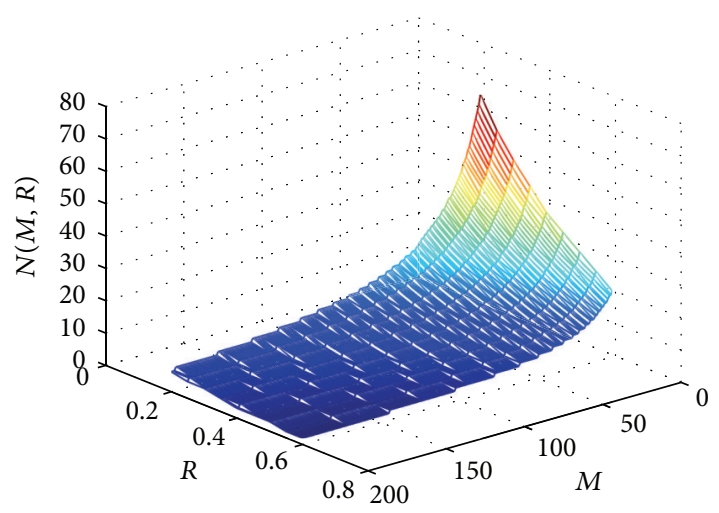

Figure 2: The amount of the failure repairment $N$ with different $M$ and $R$.

In order to discover the relationship between $M$ and $N$ with different $\mathrm{SOH}$ thresholds, $R$, which empirically ranges from 0.2 to 0.6 , and its corresponding simulation are firstly carried out and the results are illustrated in Figure 2.

Considering the long-run average cost per unit time $C(M, N)$ according to the battery management optimizing policy $(M, N)$, the amounts of premaintenance and failure repairment as well as their correspondence relations are gained from the above simulation. Assume the $\mathrm{SOH}$ replacement threshold of the battery is 0.4 ; that is, $R=0.4$; then the average costs per unit time corresponding to the different amounts of premaintenance and failure repairment are shown in Figure 3.

Due to the fact that the failure repairment cost is much more than the cost of the premaintenance, the higher average cost per unit time appears at the point of the lower value of $M$ but higher value of $N$. And the lower average cost per unit time appears mostly at the point of higher value of $M$ but lower value of $N$. Meanwhile, for the decline of the battery's $\mathrm{SOH}$ is presented in the process of repeated charging and discharging, the operating time of the model is a deteriorating system while the premaintenance time and failure repairment time are improving systems. The average cost per unit time declines at first and then increases gradually. So the lowest average cost per unit time exists and the lowest value is at the point where $\left(M^{*}, N^{*}\right)=(14,4)$, as is shown in Table 3 .

The optimal value of the average cost per unit time is further calculated as in Table 3. The following simulation is to verify if the availability corresponding to the selected point

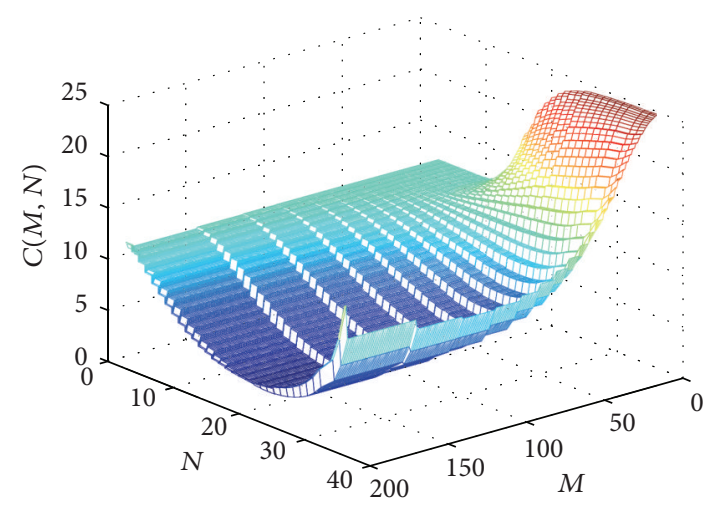

FIGURE 3: The average costs per unit time corresponding to the different $M$ and $N$ with $R=0.4$.

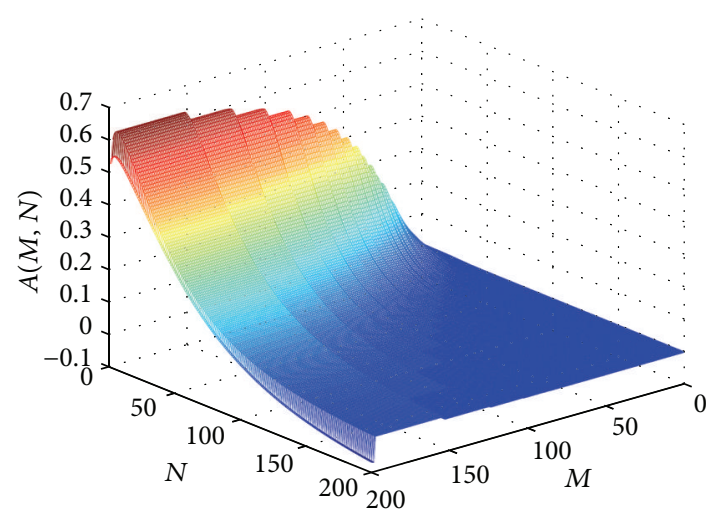

Figure 4: The availabilities with different $M$ and $N$.

$\left(M^{*}, N^{*}\right)=(14,4)$ meets the proposed threshold $A_{0}$, which is set to be 0.5 according to the empirical statistics. Under the same conditions of the previous simulations, the availabilities in the different values of $M$ and $N$ are shown in Figure 4.

As is indicated in Figure 4, in the deteriorating system of the operating time, the availability declines with the increasing of the failure repairment times in a certain amount of premaintenance, which fits the entire trend of the availability and displays a strong consistency as is shown in Figure 4. The exact values corresponding to Table 3 are shown in Table 4, where the optimized value that meets the 0.5 availability threshold is $A(14,4)=0.567$.

\section{Conclusions}

Oriented towards the batteries' deteriorating characteristics after times of repairment and the demand of the BSS profit maximization while ensuring its battery availability, this paper proposes a GP-based E-bus battery management 
TABLE 3: Average cost per unit time when $R=0.4$.

\begin{tabular}{lccccccccccc}
\hline$M$ & & & & \multicolumn{3}{c}{$N$} & & & & \\
& 36 & 23 & 19 & 16 & 12 & 9 & 6 & 5 & 11.243 \\
\hline 2 & 24.806 & 22.592 & 20.357 & 17.928 & 14.061 & 11.418 & 10.098 & 10.323 & 14.532 \\
6 & 24.697 & 21.454 & 18.379 & 15.241 & 10.572 & 7.403 & 5.155 & 4.767 & 4.709 & 6.250 \\
10 & 24.490 & 20.560 & 17.420 & 14.430 & 10.075 & 6.961 & 4.374 & 3.734 & 3.303 & 4.253 \\
14 & 24.079 & 19.474 & 16.556 & 13.976 & 10.230 & 7.335 & 4.561 & 3.744 & $3.058^{*}$ & 3.635 \\
16 & 23.752 & 18.860 & 16.126 & 13.786 & 10.372 & 7.618 & 4.797 & 3.908 & 3.118 & 3.545 \\
24 & 21.125 & 16.270 & 14.540 & 13.135 & 10.930 & 8.799 & 6.025 & 4.956 & 3.857 & 3.818 \\
28 & 18.976 & 15.124 & 13.903 & 12.879 & 11.150 & 9.309 & 6.655 & 5.548 & 4.352 & 4.141 \\
32 & 16.704 & 14.192 & 13.392 & 12.671 & 11.327 & 9.746 & 7.246 & 6.129 & 4.866 & 4.516 \\
36 & 14.819 & 13.489 & 13.000 & 12.506 & 11.468 & 10.116 & 7.786 & 6.678 & 5.377 & 4.918 \\
44 & 12.802 & 12.644 & 12.497 & 12.280 & 11.669 & 10.687 & 8.704 & 7.657 & 6.342 & 5.742 \\
\hline
\end{tabular}

${ }^{*}$ The lowest average cost per unit time.

TABLE 4: Battery availability when $R=0.4$.

\begin{tabular}{lccccccccccc}
\hline$M$ & & & & \multicolumn{3}{c}{$N$} & & & & \\
& 36 & 23 & 19 & 16 & 12 & 9 & 6 & 4 & 0.518 \\
\hline 2 & 0.005 & 0.067 & 0.131 & 0.205 & 0.330 & 0.432 & 0.529 & 0.559 & 0.588 \\
6 & 0.005 & 0.074 & 0.148 & 0.229 & 0.361 & 0.463 & 0.558 & 0.587 & 0.616 & 0.544 \\
10 & 0.005 & 0.067 & 0.132 & 0.202 & 0.322 & 0.424 & 0.528 & 0.563 & 0.597 & 0.531 \\
14 & 0.004 & 0.058 & 0.109 & 0.167 & 0.271 & 0.370 & 0.485 & 0.525 & $0.567^{*}$ & 0.509 \\
16 & 0.004 & 0.052 & 0.098 & 0.150 & 0.246 & 0.343 & 0.462 & 0.505 & 0.550 & 0.496 \\
24 & 0.003 & 0.032 & 0.059 & 0.092 & 0.162 & 0.246 & 0.371 & 0.423 & 0.480 & 0.442 \\
28 & 0.003 & 0.023 & 0.044 & 0.070 & 0.130 & 0.207 & 0.330 & 0.384 & 0.446 & 0.414 \\
32 & 0.002 & 0.016 & 0.032 & 0.053 & 0.104 & 0.174 & 0.293 & 0.348 & 0.413 & 0.387 \\
36 & 0.001 & 0.011 & 0.023 & 0.039 & 0.083 & 0.145 & 0.259 & 0.315 & 0.381 & 0.360 \\
44 & 0.000 & 0.005 & 0.011 & 0.022 & 0.052 & 0.102 & 0.202 & 0.256 & 0.324 & 0.309 \\
\hline
\end{tabular}

${ }^{*}$ The availability corresponding to the lowest average cost per unit time.

optimizing policy on condition that the battery, after premaintenance and failure repairment, cannot be as good as new. In addition, a deteriorating system is modeled for the operating time and an improving system is modeled for the premaintenance time as well as failure repairment time. The application of the GP to the battery management optimization reveals a good consistency and well reflects the operation demand of BSS. Moreover, the simulation is carried out for the analysis of the relationship between the amounts of premaintenance and failure repairment with different $\mathrm{SOH}$ thresholds when replacing the battery. Then the optimization of the long-run average cost is taken by importing those values. Finally, the simulation with the required availability threshold is carried out to verify its effectiveness. The results denote that the proposed management optimizing policy will prolong the life-cycle of the batteries and reduce the long-run average cost on the premise of the high availability, which is of much applicability on the batteries' optimizing management. And the future work is to apply the proposed GP-based Ebus battery management optimizing policy to the BSS and the microgrid to study the total cost aiming at the massive access of the E-buses and the EVs.

\section{Nomenclature}

E-bus:

GP-based:

SOC:

$\mathrm{SOH}$ :

$c_{m}$ :

$c_{f}:$

$c_{r}:$

$c_{b}:$

$c_{N}:$

$c_{c}:$

$w_{1}$ :

$w_{2}:$

$w_{3}:$

$A(M, N)$ :

$A_{0}$ :

$C(M, N)$ :
Electric bus

Geometric-process-based

State of charge

State of health

Premaintenance cost rate

Failure repairment cost rate

Replacement cost rate

Benefit rate

New battery cost

Depleted battery recycling benefit

Depreciation rate of the battery

after a single operation

Recovery rate after a single

premaintenance

Descent rate after the failure repairment

Battery availability

Threshold of the battery

availability

Long-run average cost per unit time 


$\begin{array}{ll}H: & \text { Replacement time } \\ M: & \begin{array}{l}\text { Amount of the premain- } \\ \text { tenance before one failure } \\ \text { repairment }\end{array} \\ N: & \begin{array}{l}\text { Failure repairment amount } \\ \text { during the battery life-cycle }\end{array} \\ & \text { Initial value of SOH } \\ R_{0}: & \text { Threshold of SOH } \\ R: & \begin{array}{l}\text { Life-cycle of the E-bus } \\ \text { battery } \\ T:\end{array} \\ \left\{X_{n}^{i}, n=1,2, \ldots, i=1,2, \ldots\right\}: \begin{array}{l}\text { Battery operating time after } \\ \text { the } i \text { th premaintenance in }\end{array} & \text { the } n \text {th period } \\ \left\{Y_{n}^{i}, n=1,2, \ldots, i=1,2, \ldots\right\}: & \begin{array}{l}\text { The } i \text { th premaintenance } \\ \text { time in the } n \text {th period }\end{array} \\ \left\{Z_{n}, n=1,2, \ldots\right\}: & \text { Failure repairment time in } \\ & \text { the } n \text {th period. }\end{array}$

\section{Conflict of Interests}

The authors declare that there is no conflict of interests regarding the publication of this paper.

\section{Acknowledgments}

This work is supported by the National Natural Science Foundation of China under Grants nos. 61374097 and 201202073 and the Program for New Century Excellent Talents in University (no. NCET-12-0103).

\section{References}

[1] P. Han, J. Wang, Y. Han, and Y. Li, "Resident Plug-In Electric Vehicle charging modeling and scheduling mechanism in the smart grid," Mathematical Problems in Engineering, vol. 2014, Article ID 540624, 8 pages, 2014.

[2] K. Rajashekara, "Present status and future trends in electric vehicle propulsion technologies," IEEE Journal of Emerging and Selected Topics in Power Electronics, vol. 1, no. 1, pp. 3-10, 2013.

[3] Y. Li, J.-K. Wang, P. Han, and Y.-H. Han, "Modeling and analysis on coordinated scheduling of E-bus recharging station participated wind-power generation," in Proceedings of the 32nd Chinese Control Conference (CCC '13), pp. 8592-8596, Xian, China, July 2013.

[4] Q. Dai, T. Cai, S. Duan, and F. Zhao, "Stochastic modeling and forecasting of load demand for electric bus battery-swap station," IEEE Transactions on Power Delivery, vol. 29, no. 4, pp. 1909-1917, 2014.

[5] P. Elbert, T. Nuesch, A. Ritter, N. Murgovski, and L. Guzzella, "Engine on/off control for the energy management of a serial hybrid electric bus via convex optimization," IEEE Transactions on Vehicular Technology, vol. 63, no. 8, pp. 3549-3559, 2014.

[6] C. R. Gould, C. M. Bingham, D. A. Stone, and P. Bentley, "New battery model and state-of-health determination through subspace parameter estimation and state-observer techniques," IEEE Transactions on Vehicular Technology, vol. 58, no. 8, pp. 3905-3916, 2009.

[7] V. Agarwal, K. Uthaichana, R. A. Decarlo, and L. H. Tsoukalas, "Development and validation of a battery model useful for discharging and charging power control and lifetime estimation,"
IEEE Transactions on Energy Conversion, vol. 25, no. 3, pp. 821$835,2010$.

[8] Y. Wang and Y. Liu, "Electronic control system design and test of pure electric vehicle battery management system," in Proceedings of the 2nd International Conference on Mechanic Automation and Control Engineering (MACE '11), pp. 1289-1292, IEEE, Hohhot, China, July 2011.

[9] H. Y. Li, Y. L. Jia, D. Zhang et al., "Application of electric vehicle battery intelligent monitoring and management system," in Proceedings of the IEEE Conference and Expo Transportation Electrification Asia-Pacific, pp. 1-5, Beijing, China, 2014.

[10] H. C. Han, H. X. Xu, Z. Q. Yuan, and Y. Shen, "A new SOH prediction model for lithium-ion battery for electric vehicles," in Proceedings of the 17th International Conference on Electrical Machines and Systems (ICEMS '14), pp. 997-1002, Hangzhou, China, October 2014.

[11] M. Paul-Henri and H. Vincent, "An adaptive sigma point kalman filter hybridized by support vector machine algorithm for battery SoC and SoH estimation," in Proceedings of the IEEE 81st Vehicular Technology Conference, pp. 1-7, Glasgow, UK, May 2015.

[12] L.-R. Dung, S.-H. Wu, and H.-F. Yuan, "An SOH estimation system based on time-constant-ratio measurement," in Proceedings of the IEEE 23rd International Symposium on Industrial Electronics (ISIE '14), pp. 1784-1787, Istanbul, Turkey, June 2014.

[13] Y. Lam and Y. L. Zhang, "A geometric-process maintenance model for a deteriorating system under a random environment," IEEE Transactions on Reliability, vol. 52, no. 1, pp. 83-89, 2003.

[14] L. Tan, T. Cheng, and B. Guo, "Optimal maintenance strategy for the repairable system consisting of one component based on geometric process," Systems Engineering, vol. 26, no. 6, pp. 88-92, 2008.

[15] J. Jia, D. Wang, and Z. Duan, "Study on the mathematical model under optimal replacement for a deteriorative repairable system," Mathematics in Practice and Theory, vol. 36, no. 4, pp. $1-4,2006$.

[16] Y. Lam, "A geometric process $\delta$-shock maintenance model," IEEE Transactions on Reliability, vol. 58, no. 2, pp. 389-396, 2009.

[17] G. J. Wang and Y. L. Zhang, "A bivariate optimal replacement policy for a cold standby repairable system with preventive repair," Applied Mathematics and Computation, vol. 218, no. 7, pp. 3158-3165, 2011.

[18] Y. L. Zhang and G. J. Wang, "A geometric process repair model for a series repairable system with k dissimilar components," Applied Mathematical Modelling, vol. 31, no. 9, pp. 1997-2007, 2007.

[19] Y. L. Zhang and G. J. Wang, "An extended replacement policy for a deteriorating system with multi-failure modes", Applied Mathematics and Computation, vol. 218, no. 5, pp. 1820-1830, 2011.

[20] G. J. Wang and Y. L. Zhang, "Optimal periodic preventive repair and replacement policy assuming geometric process repair," IEEE Transactions on Reliability, vol. 55, no. 1, pp. 118-122, 2006.

[21] Y. L. Zhang, "A geometric-process repair-model with good-asnew preventive repair," IEEE Transactions on Reliability, vol. 51, no. 2, pp. 223-228, 2002.

[22] Y. Han, J. Wang, Q. Zhao, and P. Han, "An optimal operating strategy for battery life cycle costs in electric vehicles," Journal of Applied Mathematics, vol. 2014, Article ID 305905, 6 pages, 2014. 


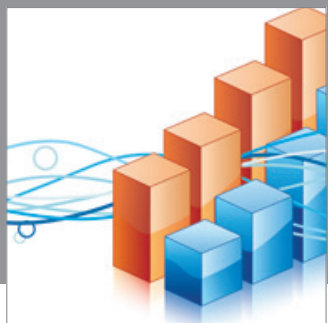

Advances in

Operations Research

mansans

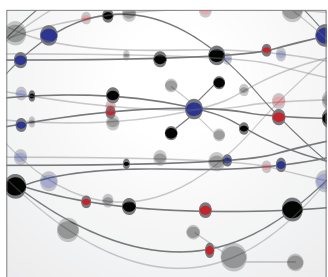

The Scientific World Journal
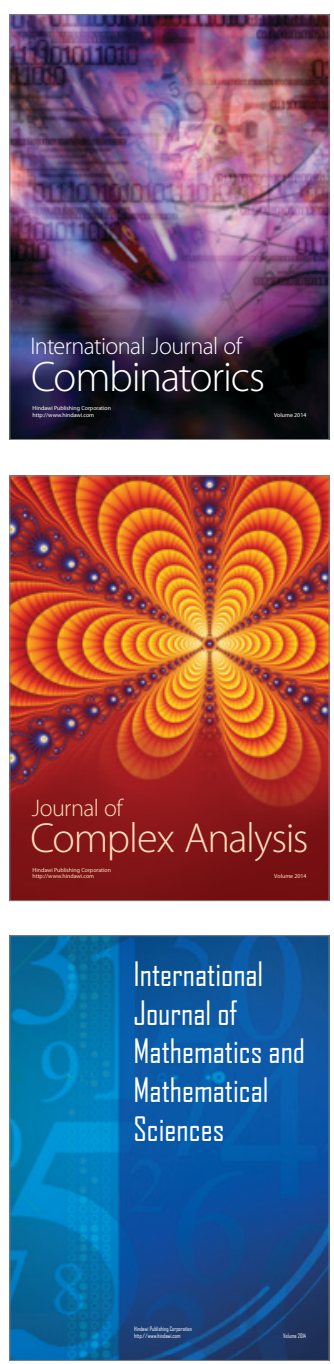
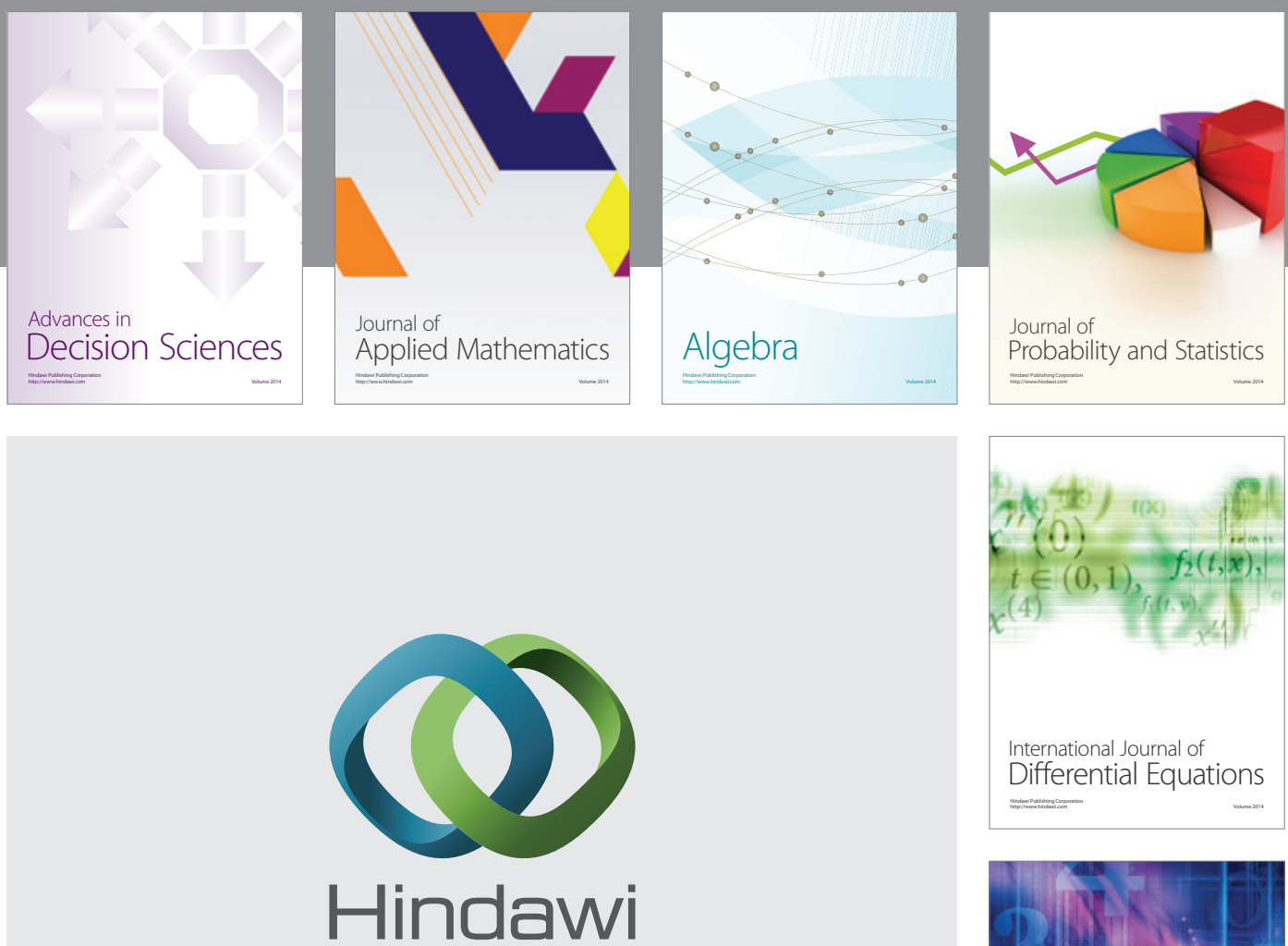

Submit your manuscripts at http://www.hindawi.com
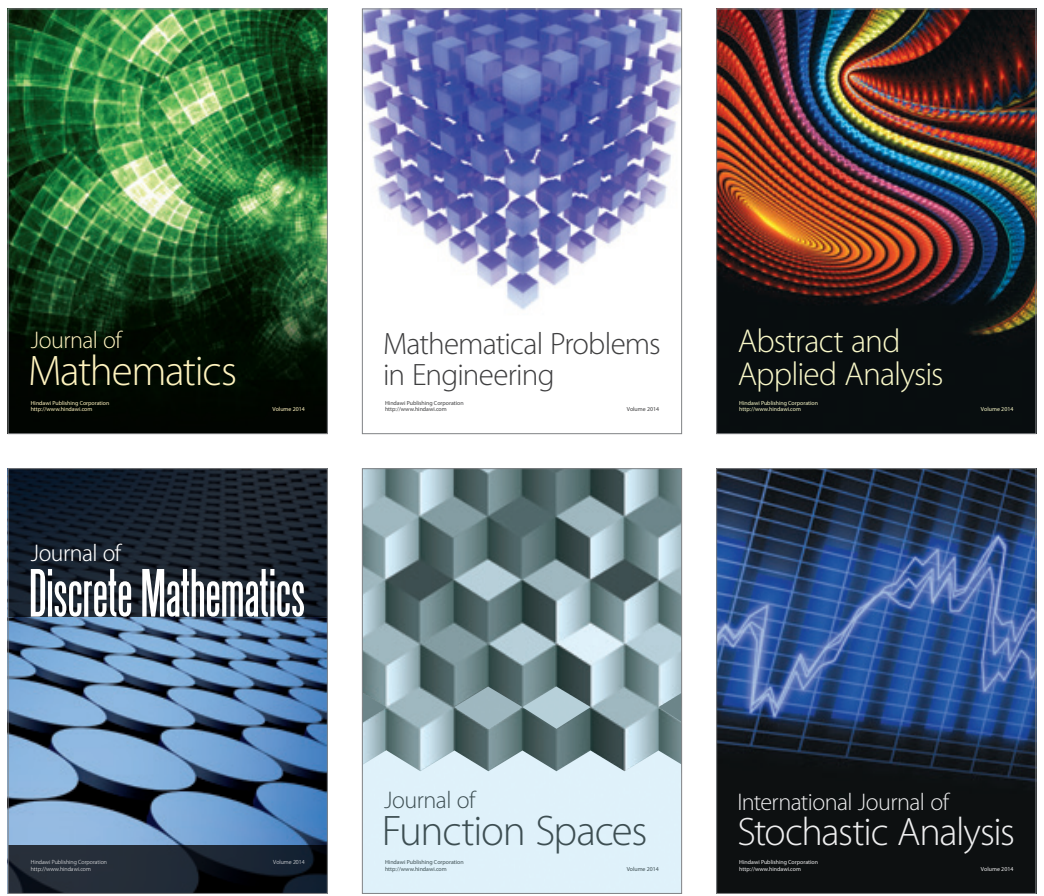

Journal of

Function Spaces

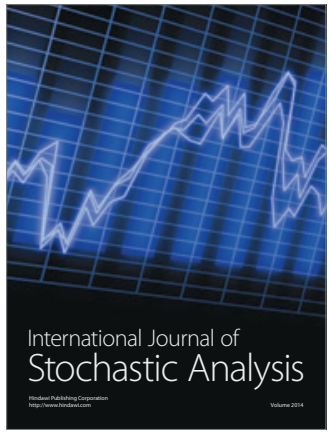

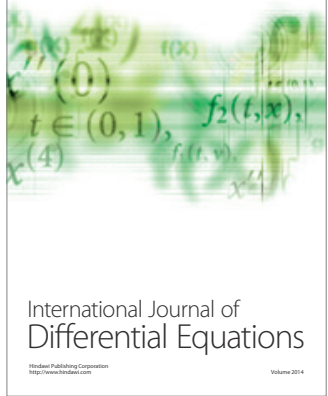
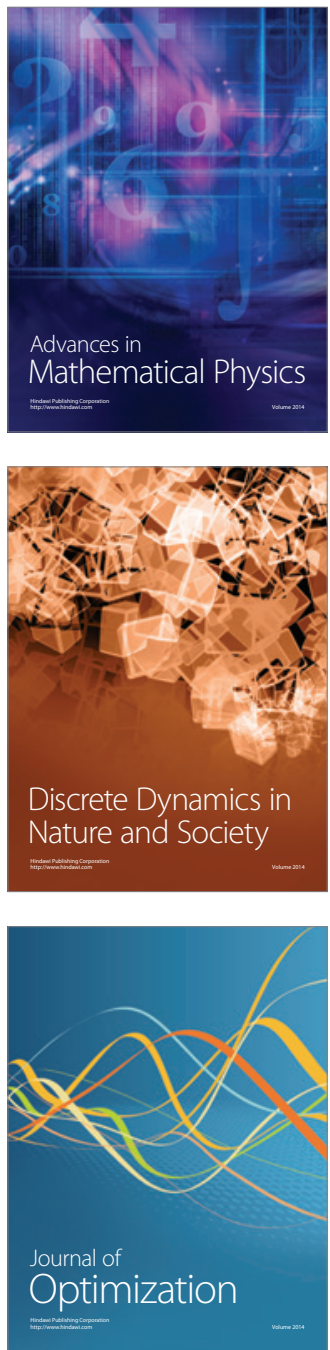